OPEN ACCESS

Edited by:

Margarida Matos,

University of Lisbon, Portugal

Reviewed by:

Shaoqing Tang,

Guangxi Normal University, China Jian-Feng Mao,

Beijing Forestry University, China

*Correspondence:

Yuguo Wang

wangyg@fudan.edu.cn

Specialty section:

This article was submitted to Evolutionary and Population Genetics,

a section of the journal

Frontiers in Genetics

Received: 03 May 2019 Accepted: 13 November 2019

Published: 04 February 2020

Citation:

Yang J, Fu W, Xu H, Song Z, Zhang W,

Yang $J$ and Wang $Y$ (2020) An Empirical Analysis Rejects the Hybrid Speciation Hypothesis of a Crucial Kiwifruit Species, Despite Genomic Evidence of Frequent Interspecific

Gene Flow in the Genus.

Front. Genet. 10:1250

doi: 10.3389/fgene.2019.01250

\section{An Empirical Analysis Rejects the Hybrid Speciation Hypothesis of a Crucial Kiwifruit Species, Despite Genomic Evidence of Frequent Interspecific Gene Flow in the Genus}

\author{
Jie Yang, Weirui Fu, Haoming Xu, Zhiping Song, Wenju Zhang, Ji Yang and Yuguo Wang * \\ Ministry of Education Key Laboratory for Biodiversity Science and Ecological Engineering, Institute of Biodiversity Science, \\ School of Life Sciences, Fudan University, Shanghai, China
}

Hybrid speciation is an important way to generate species diversity. In general, however, interspecific hybridization is easily confused with the formation of hybrid species. Using the genomic resequencing data of the kiwifruit genus (Actinidia), at least ten species were documented recently as homoploid hybrid species, and thus a two-layer mode of species diversification has been proposed. As a crucial piece of evidence, Actinidia fulvicoma was identified as a hybrid derivative of Actinidia eriantha $\times$ Actinidia cylindrica, representing a rare case of hybrid species in kiwifruit that won the competition of ecological niches with one of its putative parental species, $A$. cylindrica. However, the hypothesized hybrid origin of $A$. fulvicoma is inconsistent with our specimen observations. Here, we present multiple lines of evidence to reject the hybrid speciation hypothesis for this species, despite genomic evidence for frequent interspecific gene flow. We collected the samples of $A$. fulvicoma in type locality and neighboring regions to contrast them with type specimen, and sequenced nuclear ribosomal DNA ITS, chloroplast trnL-trnF and mitochondrial nad2-i3, as well as four single-copy nuclear genes explored from kiwifruit genomes, to infer phylogenetic relationships among $A$. fulvicoma, its putative parental species, and their relatives. Our data definitely reveal that $A$. fulvicoma occupies an independent backbone lineage and it is not a hybrid. This study suggests that correct evolutionary applications on extensive surveys of the putative hybrid and its possible parents with strict criteria are necessary in the documentation of hybrid speciation to advance our understanding of the genomic basis of hybrid species.

Keywords: kiwifruit, interspecific hybridization, Actinidia fulvicoma, hybrid speciation, species identification

"Surprisingly, although there is widespread establishment of allopolyploid hybrids in Actinidia, homoploid hybrid speciation is prevalent in this genus... The hybrid A. fulvicoma var. fulvicoma should be a winner in the competition for ecological niches because one of its parents, A. cylindrica, has a very restricted distribution within its wide geographic range, suggesting that hybridization can widen ecological adaptation." (Liu et al., 2017, p. 886) 


\section{INTRODUCTION}

As one of the important evolutionary forces, natural hybridization occurs frequently in flowering plants. It is estimated that an average of $25 \%$ of plant species are known to hybridize with at least one other species (Mallet, 2005). Interspecific hybrids from distantly related parent species are often sterile or inviable (Mayr, 1963; Coyne and Orr, 2004). Even if a healthy hybrid is formed, the offspring usually backcrosses to the more abundant parent species rather than going through selffertilization, and will therefore often be unfit (Mallet, 2007). The fact that most plant hybrid zones are limited to an extent implies that hybrids are on average less fit than their parents, at least in parental habitats (Coyne, 1996; Rieseberg, 1997). However, in a few cases, the reproductive barrier between a few hybrid offspring and their parents were built up, and, eventually, the offspring could reproduce independently to form a new hybrid linage. For example, two diploid species of Helianthus ( $H$. annuus and $H$. petiolaris) produced three diploid species $(H$. anomalus, $H$. deserticola, and $H$. paradoxus) through ancient hybridization (Rieseberg et al., 1995; Gross and Rieseberg, 2005). These hybrids are distributed in deserts, salt-rich swamps, or other suitable open habitats that are not occupied by parent species, respectively. Although interspecific hybridization in flowering plants is relatively common, tests of hybrid speciation hypotheses using phylogenetic approaches have rejected such hypotheses many times (e.g. Rieseberg and Wendel, 1993; Wolfe and Elisens, 1993; Wolfe and Elisens, 1994; Wolfe and Elisens, 1995; Arnold, 1997). Only a few cases of homoploid hybrid speciation have been documented when strict criteria are applied (Schumer et al., 2014). Despite the argument of whether these criteria are too stringent (Feliner et al., 2017; Schumer et al., 2018), there is no doubt that many putative hybrid species need to be re-tested.

Owing to frequent interspecific hybridization, the kiwifruit genus (Actinidia Lindl.) has been recognized as an ideal material for studying hybrid speciation. The distribution areas of wild kiwifruit species often overlap, and hybridization between taxa is common in nature (Huang and Liu, 2014). The Actinidia species are well documented for the contrasting mode of the maternal inheritance of mitochondrial $(\mathrm{mt})$ genes and paternal inheritance of most chloroplast (cp) genes (Cipriani et al., 1995; Testolin and Cipriani, 1997; Chat et al., 1999; Li et al., 2013), and, like other flowering plants, their nuclear genes biparentally inherit. Through examining the phylogenetic incongruences among different kinds of genes such as nuclear ribosomal (nr) DNA ITS vs. cp gene matK (Li et al., 2002), mtDNA vs. cpDNA (Chat et al., 2004), and nuclear markers vs. cytoplasmic DNA sequences (Li et al., 2007), several taxa were identified as the putative interspecific hybrids in Actinidia.

Recently, the history of reticulate evolution of Actinidia was reconstructed through genomic DNA sequencing of 25 taxa, and the hybrid origins of at least ten species were documented (Liu et al., 2017). Based on these findings, a two-layer mode of species diversification was further proposed, and homoploid hybrid speciation was particularly emphasized as an important representative mechanism fueling rapid reticulate radiations.
Unexpectedly, like A. cylindrica var. reticulata, A. fulvicoma var. fulvicoma was also documented as a hybrid species produced by A. cylindrica and A. eriantha, whereas the variety of this species, A. fulvicoma var. hirsuta, is not a hybrid (Liu et al., 2017). It is worth noting that the distribution of A. cylindrica is limited to a few counties in Guangxi, China, while A. fulvicoma var. fulvicoma is widely distributed in several provinces in southern China. They further concluded that the wide distribution of A. fulvicoma var. fulvicoma relative to A. cylindrica was the result of ecological differentiation promoted by hybridization.

Interspecific hybridization has proven to be the ecological differentiation of species (e.g., Rieseberg et al., 2003) and even the expansion of distribution areas of hybrids in some cases of homoploid hybrid speciation (Hovick and Whitney, 2014), but such special cases, like A. fulvicoma var. fulvicoma as mentioned above, are rare. Judging from voucher photographs of this species of Liu et al. (2017), however, it is inconsistent with our observations. The sample of A. fulvicoma of Liu et al. (2017) has pink flowers with seven petals, orange anthers, and gray fruit without persistent sepals. These characteristics do not completely match the original description of A. fulvicoma: white flower with five petals, yellow anthers, and dark green fruit with persistent sepals folded back. These morphological comparisons imply that it is likely that incorrect identification or a wrong sampling of $A$. fulvicoma led to error conclusion of its hybrid origin in the study of Liu et al. (2017).

To verify whether A. fulvicoma is of hybrid origin, we collected population samples from its type locality, Mount Luofu, Guangdong, and its adjacent areas. Phylogenetic analyses and mutation statistics based on multiple genes with different inherited modes, specimen comparisons involving type specimens and voucher photographs of Liu et al. (2017), and a geographic distribution analysis of A. fulvicoma and its putative parental species inferred by Liu et al. (2017) were produced to address whether interspecific hybridization occurred. However, our evidence does not support that A. fulvicoma is a hybrid species between A. cylindrica and A. eriantha.

\section{MATERIALS AND METHODS}

We present here a practical method to assess whether a species is of hybrid origin, and it involves DNA sampling, gene amplification, sequence analysis, and the comparison of distribution and morphology. We reason that if a taxon is not of hybrid origin, the samples collected from the type locality or neighboring areas would occupy independent lineages apart from putative parental species in both nuclear and cytoplasmic gene trees, or, if there exist more distinct variable mutations between the species and any putative parental species, it should not be a hybrid taxa. Similarly, in this case, the geographical distribution of the two putative parental species would not overlap. On the contrary, the nuclear genes of the hybrid species would contain genetic components of both parents, while the sequences of its cytoplasmic genes would be similar to those of one parent. In our notation, its distribution should be within, or not far from, the overlapping regions of two parent species. 


\section{Sample Collection and DNA Extraction}

The samples of A. fulvicoma and its varieties were collected from its type locality (Mount Luofu) and neighboring areas (Nanxiong and Mount Nankun) in Guangdong, China, and other localities such as Mount Daoyao and Nandan in Guangxi, China (Table S1). The two putative parental species ( $A$. eriantha and $A$. cylindrica) and other Actinidia species were collected form the Guilin Botanical Garden, Guangxi, or from the field. All voucher specimens were deposited in the Herbarium of Fudan University (FUS). The species taxonomy primarily follows the classification of Actinidia in Flora Republicae Popularis Sinicae (i.e. the Chinese version of Flora of China, Liang et al., 1984).

The fresh young leaves were dried with silica gel and the total DNA of each sample was isolated using a Tiangen Plant Genomic DNA Kit (Tiangen Biotech Co., Beijing, China) following the manufacturer's instructions. The quality of extracted genomic DNA was assessed by agarose gel electrophoresis.

\section{PCR Amplification and Gene Sequencing}

The amplification and sequencing primers of the ITS gene were ITS4 and ITS5 (White et al., 1990). Primers c and f (Taberlet et al., 1991) were used as the amplification and sequencing primers of cpDNA $\operatorname{trn} \mathrm{L}-\operatorname{trnF}$. The mtDNA nad2-i3 sequences were amplified in two segments by the nested PCR method. Five primers $(769 \mathrm{~F}, 813 \mathrm{~F}, 2 \mathrm{~F}, 881 \mathrm{R}$, and $1227 \mathrm{R})$ were newly designed. The first set of primers were $1 \mathrm{R}$ (Cipriani et al., 2003), 769F (5'-GCGAAGAGAATAAGATGCTGC-3'), 2F (5'TGAAATCACTGGTGCTCG-3'), and 1277R (5' GCCTTTCCTTGAATGGTTG-3'). The first PCR amplification products were used as the DNA template for the second PCR. Primers 1R, 813F (5'-CCAGAATAAGATGCTGCTCC-3'), 2F, and $881 \mathrm{R}$ ( $5^{\prime}$-GCCTTAGAGGGGAAGATTAGC-3') were used as the second set of PCR primers. The primers $1 \mathrm{R}, 813 \mathrm{~F}$, and $881 \mathrm{R}$ were used for cycle sequencing. Because high polymorphism, pseudogenization, and non-concerted evolution of nrDNA ITS occurs in some flowering plant genera such as Quercus (Mayol and Rosselló, 2001) and Pyrus (Zheng et al., 2008), we also needed to test whether A. fulvicoma was a real interspecific hybrid from $A$. eriantha and $A$. cylindrica using other nuclear genes. Therefore, new primers were specially designed to amplify and sequence four single-copy nuclear genes (SCNGs) from different chromosomes (Table S2) according to the sequences of diploid genome of $A$. chinensis cv. Hongyang (Huang et al., 2013).

Polymerase chain reactions were performed in a $50 \mu \mathrm{l}$ reaction volume containing $\sim 150 \mathrm{ng}$ of total DNA, $5 \mu \mathrm{l}$ of $10 \times$ PCR Buffer $\left(\mathrm{Mg}^{2+}\right.$ free), $6 \mu \mathrm{l}$ of $\mathrm{MgCl}_{2}$ (2.5 mmol.L-1), $8 \mu \mathrm{l}$ of dNTP Mixture (2.5 mmol.L-1), $3 \mu \mathrm{l}$ of forward primer (10 $\mu \mathrm{mol} \cdot \mathrm{L}-1), 3 \mu \mathrm{l}$ of reverse primer $(10 \mu \mathrm{mol} \cdot \mathrm{L}-1)$, and $2.5 \mathrm{unit}$ of Taq polymerase. The PCR running programs for ITS, trnLtrnF, nad2-i3, and four SCNGs are presented in Table S3. PCR products were visualized on $1.0 \%$ agarose gels and purified on 3.0\% agarose gels using a Gel Extraction System B Kit (BioDevTech, Beijing, China). Purified PCR products were sequenced directly by a BigDye Terminator 3.1 Cycle Sequencing Kit (Applied Biosystems, CA, USA) and run in an ABI PRISM 377 DNA Sequencer (Applied Biosystems, CA, USA). For PCR products possessing heterozygous sequences, they were ligated into pMD-18T vector (TaKaRa Co., Dalian, China) and cloned into plasmids. Eight separate clones with correct insert were then screened by comparing restriction fragments for cycle sequencing (Lee et al., 2002; Kim et al., 2008). All sequences generated in this study were submitted to GenBank (accession numbers: MK425065-MK425153 and MK614167-MK614214).

\section{Phylogenetic Analyses}

The sequences of our samples and other sequences obtained from GenBank were assembled by a Seqman II 5.05 (DNAStar, London, UK). The alignment of gene sequences was undertaken by a Clustal X 2.0 (Larkin et al., 2007) and checked manually. All gaps in the alignment were treated as missing data. Maximum likelihood (ML) was performed on three sets of independent sequence data. ML analyses were run in Randomized Accelerated Maximum Likelihood (RAxML) 7.0.4 (Stamatakis, 2006), and the general time reversible (GTR) model and gamma distribution were used for constructing the phylogenetic relationships of the species involved. Support values for ML trees were estimated with 1,000 bootstrap replicates. MEGA 5.1 (Tamura et al., 2011) was used for the input trees. The species of Clematoclethra or Saurauia were placed as the root of the tree. In each tree, the accession number of the sequence downloaded from GenBank was marked behind the accession name.

\section{Distribution and Morphology}

In view of the hypothesized hybrid origin of A. fulvicoma (Liu et al., 2017), we selected $A$. fulvicoma and two putative parental species (A. eriantha and A. cylindrica) to analyze their natural distributions. The geographic data from specimen records of four main herbaria (IBK, IBSC, KUN, and PE) and previous field investigations (Cui, 1993; Qing and Liu, 2010) were collected to plot the distributions and to check if the two putative parental species overlap geographically. We compared the specimens of A. fulvicoma sampled from the type locality, the neighboring areas, and other localities with type specimen, and the specimens identified by Choufen Liang, who edited Actinidia of Flora Republicae Popularis Sinicae (Liang et al., 1984), were checked to see whether A. fulvicoma has intermediate morphological characters of putative parental species, A. eriantha and A. cylindrica.

\section{RESULTS}

\section{Morphological Comparison}

For the type specimen of A. fulvicoma, deposited in the Herbarium of the Royal Botanic Gardens, Kew (K) (Figure 1A), had a leaf blade that is oblong-ovate and broadly ovate, papery; the branchlets and leaves were abaxially and densely tomentose; the fruit was oblong-ovoid; and persistent sepals reflexed. The specimens identified by C. Liang had similar characters with a little difference on the leaf blade oblong-ovate and they had brownish velutinous (Figure 1B). For the samples we collected from the type locality and adjacent areas, the leaf blade was papery, suborbicular to oblong-ovate, fruit narrowly obovate, and speckled, about $0.6-1.5 \mathrm{~cm}$, dark green, persistent sepals reflexed. The morphological characters of these samples fall into the range 


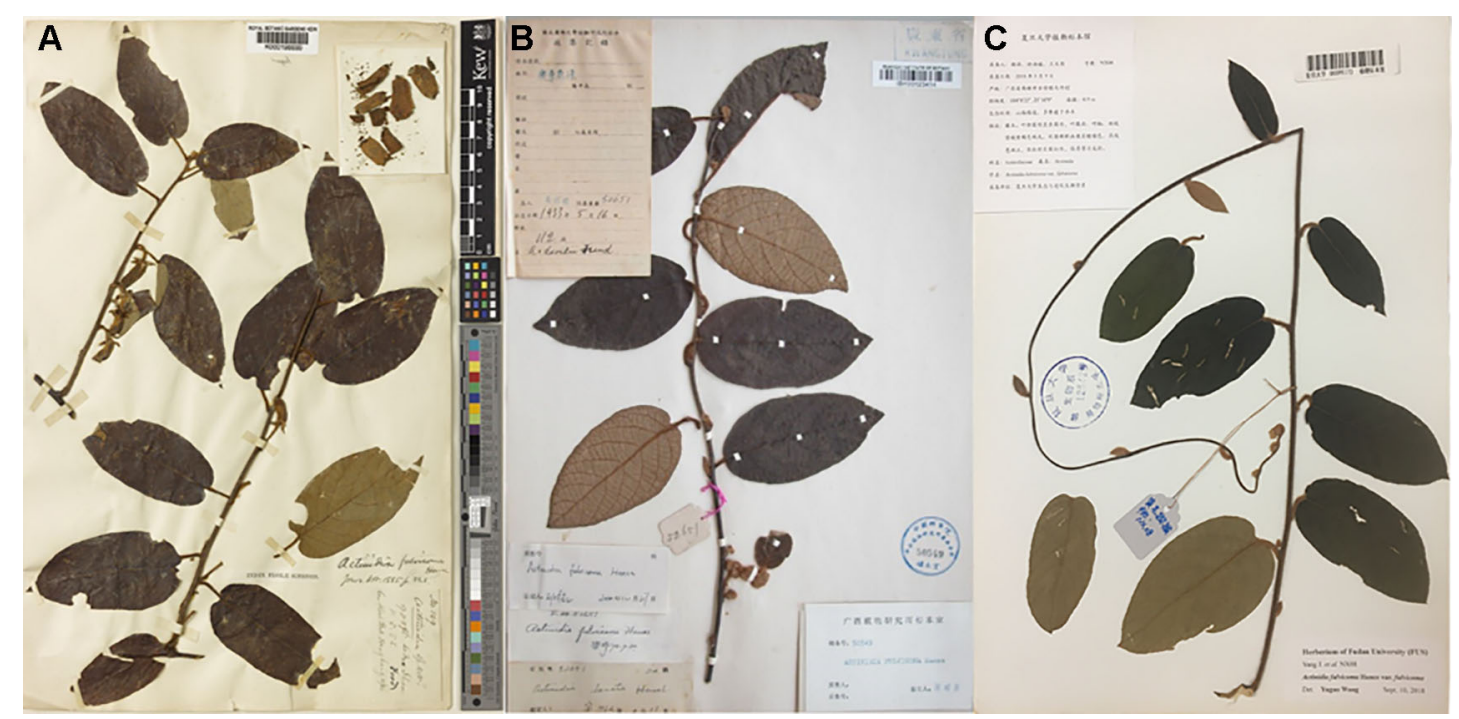

FIGURE 1 | Specimen photographs of Actinidia fulvicoma. The type specimen of A. fulvicoma deposited in the Herbarium of the Royal Botanic Gardens, Kew (A), the specimens identified by $\mathbf{C}$. Liang (B) and collected in this study (C). The leaf blade of $A$. fulvicoma in our specimen has oblong-ovate to suborbicular leaves, the leaf blade is papery, and the leaf venter and young branchlets are covered with dense and stellate tomentum, which shows almost the same morphological characters with the type specimens and the specimen of $A$. fulvicoma identified by $\mathrm{C}$. Liang.

of populations of A. fulvicoma. Although the variations exist in the wild populations, we can still find samples that are very similar to type specimens (Figure 1C). Our samples of A. fulvicoma from the type locality and adjacent areas were not similar to that of Liu et al. (2017), which voucher picture showed that its morphology was like the two putative parental species, and specially, its fruit was nearly round, close to that of $A$. eriantha [See Figure S1 of Liu et al. (2017)]. Instead, our samples of A. fulvicoma showed a high degree of similarity with their 'hirsuta' sample of A. fulvicoma, which morphological characters of leaf, flower, and fruit were clearly different to those of A. eriantha and A. cylindrica.

\section{Phylogenetic Analyses ITS Data}

The data matrix of 42 sequences from 23 species, including 24 sequences obtained from GenBank and 18 sequences generated in this study, was used to construct an ITS phylogenetic tree. The fulllength ITS sequence includes three regions, ITS1, 5.8S, and ITS2. Of the 649 nucleotide sites, 103 sites were parsimony informative after alignment. The GC content of the ITS alignment matrix was $55 \%$. Bootstrap values for maximum likelihood are presented above the branches in Figure 2. The phylogenetic tree based on ITS data obtained similar topologies of main clades within Actinidia as a previous study (Li et al., 2002). All samples of A. fulvicoma and its varieties formed a monophyletic group with 91\% maximum likelihood bootstrap (MLBS), and they did not directly cluster with either of the two putative parental species, A. eriantha or A. cylindrica. A. eriantha was sister to A. latifolia (87\% MLBS), whereas A. cylindrica clustered with A. farinosa $(57 \%$ MLBS). A. fulvicoma var. lanata was sister to other accessions of A. fulvicoma. There were seven single nucleotide polymorphisms (SNPs) between var. lanata and the common type of A. fulvicoma but only one nucleotide difference between var. lanata f. hirsuta and A. fulvicoma. There was no difference between A. fulvicoma var. fulvicoma and A. fulvicoma var. pachyphylla. However, there were obvious SNP differences among A. fulvicoma, A. eriantha, and $A$. cylindrica (Table 1). Similar results were obtained by genetic distance analysis: the genetic distance between the population samples of A. fulvicoma var. fulvicoma and A. fulvicoma var. pachyphylla was $0-0.00159$, which was among the range of all population samples of A. fulvicoma (0-0.00479); however, the genetic distance between A. fulvicoma and $A$. eriantha and between A. fulvicoma and A. cylindrica were $0.03998-0.044493$ and $0.02238-0.02566$, respectively, showing clear differences at the species level. Furthermore, there were more than two ITS haplotypes in the samples from Dayao mountain populations of A. fulvicoma isolated by molecular cloning. Phylogenetic analysis shows that they were nested within the same clade of $A$. fulvicoma and its varieties, and no sample clustered with A. eriantha or A. cylindrica (Figure S1).

\section{Mitochondrial and Chloroplast Data}

The alignment of mtDNA nad2-i3 is $1687 \mathrm{bp}$, and only 14 were parsimony informative characters with an insertion/deletion (InDel). The nad2-i3 gene data contains 35 sequences from 22 species, an all sequences were generated in this study. The GC content of the nad2-i3 alignment matrix was $47.5 \%$. Despite the poor phylogenetic resolution (MLBS $<50 \%$ ), either $A$. eriantha or A. cylindrica still formed a monophyly (Figure S2). There were four phylogenetic informative characters and a 5-bp InDel among A. fulvicoma, A. eriantha, and A. cylindrica. As shown in Table 2, there were three distinct SNP sites and a 4-bp InDel difference between the nad2-i3 nucleotide sequence of $A$. cylindrica and A. fulvicoma. A. eriantha showed a nucleotide sequence that was similar to seven individuals of $A$. fulvicoma, with the exception of a difference in $\mathrm{A} / \mathrm{G}$ at site 1032 . 


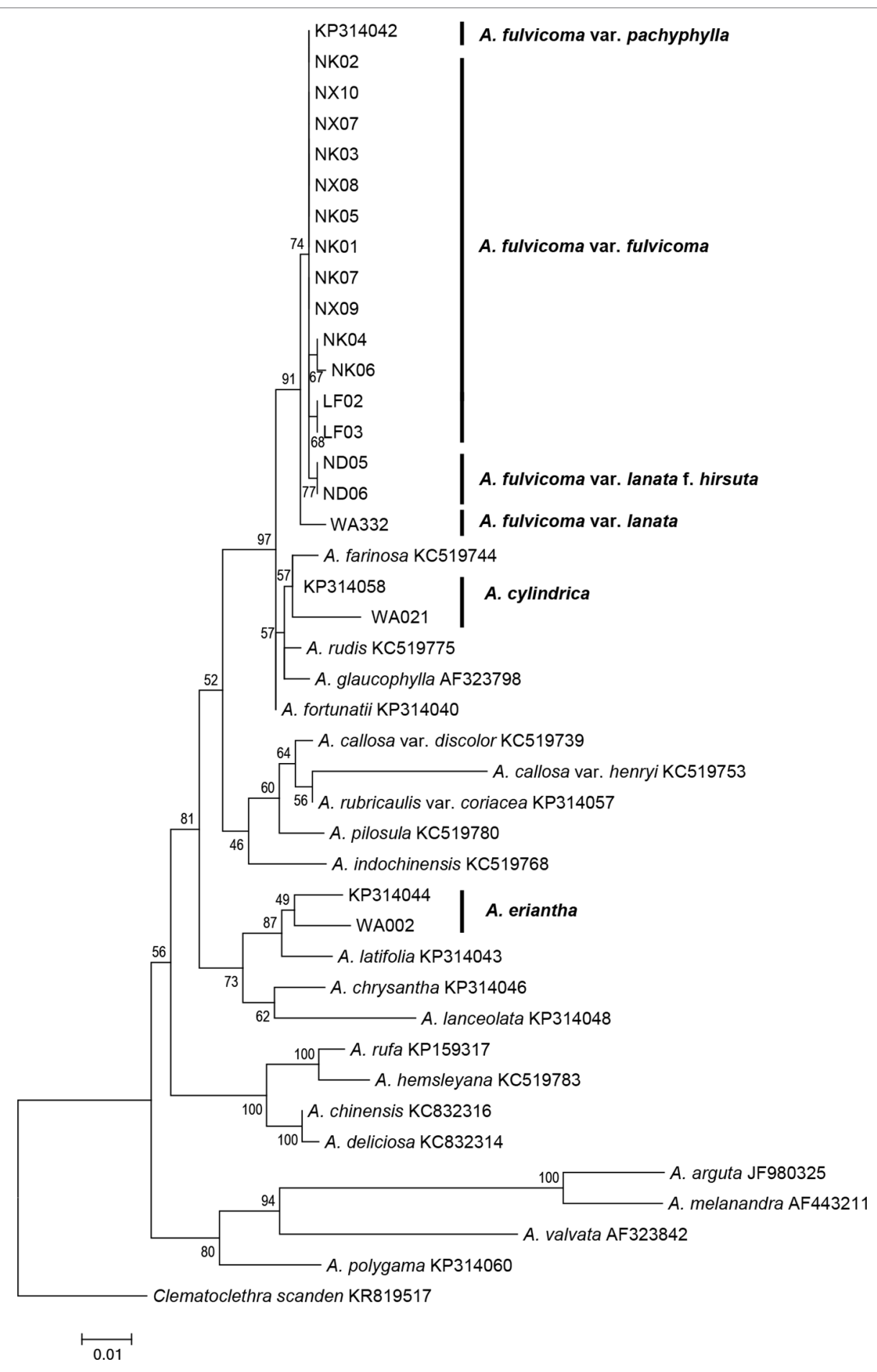

FIGURE 2 | Phylogenetic analysis of Actinidia species based on ITS sequences using RAxML. All the samples of $A$. fulvicoma and its varieties form a clade. The ITS tree shows that $A$. fulvicoma is distantly related with the putative parental species $A$. eriantha and $A$. cylindrica, which means that $A$. eriantha and $A$. cylindrica are not the putative parental species of $A$. fulvicoma. Numbers above the branch are bootstrap values result from 1,000 replicates.

The alignment length of cpDNA trnL-trnF was $926 \mathrm{bp}$; it contained nine parsimony informative characters as well as two InDels. The $\operatorname{trn} \mathrm{L}-\operatorname{trn} \mathrm{F}$ gene data include 33 sequences from 15 species, and 11 sequences were obtained from GenBank and 22 sequences were generated in this study. The GC content of the $\operatorname{trn} \mathrm{L}-\operatorname{trn} \mathrm{F}$ alignment matrix was $33.9 \%$. In the $\operatorname{trn} \mathrm{L}-\operatorname{trn} \mathrm{F}$ tree, $A$. fulvicoma clustered with A. cylindrica, but the accessions of $A$. fulvicoma did not form a monophyly (Figure S3). There was one $\mathrm{C} / \mathrm{T}$ mutations at site 271 and a $\mathrm{T}$ insert at sites $284-285$ between A. fulvicoma and A. cylindrica, but there were also some 
TABLE 1 | The variable sites of nrDNA ITS sequences.

\begin{tabular}{rr}
\hline Absolution position & 1112222222444455555555555566 \\
2237388890190022344358811356777889902 \\
1593156966922312916834578507358270917
\end{tabular}

\section{A. fulvicoma var. fulvicoma}

NX07 TCTTCTGTTGTTATTCCTGGCTT - - ACTC- CGTTTTT NX08 TCTTCTGTTGTTATTCCTGGCTT - - ACTC- CGTTTT -

NX09 TCTTCTGTTGTTATTCCTGGCTT - - ACTC- CGTTTTT NX10 TCTTCTGTTGTTATTCCT GGCTT - - ACTC- CGTTTTT NK01 TCTTCTGTTGTTATTCCT GGCTT - - ACTC- CGTTTTT NKO2 TCTTCTGTTGTTATTCCTGGCTT - - ACTC- CGTTTTT NKO3 TCTTCTGTTGTTATTCCTGGCTT - - ACTC- CGTTTT NKO4 TTTTCTGTTGTTATTCCTGGCTT - - ACTC- CGTTTTT NK05 TCTTCTGTTGTTATTCCTGGCTT - - ACTC- CGTTTTT NK06 TTTTCTGTTGTTATTCCTGGCTT- - ACTC- CGTTTTG NK07 TCTTCTGTTGTTATTCCTGGCTT - - ACTC- CGTTTTT LFO2 TCTTCTGTTGTTATTCATGGCTT- - ACTC- CGTTTTT LFO3 TCTTCTGTTGTTATTCATGGCTT - - ACTC- CGTTTTT A. fulvicoma var. pachyphylla

KP314042 TCTTCTGTTGTTATTCCTGGCTT - - ACT C- CGTTTTT A. fulvicoma var. lanata

WA332

TCATCT G- TCTTACT CCT GGCTTTTATTC- CGTTTTT

A. fulvicoma var. lanata f. hisuta

ND05

ND06

TCTTCT GTTGTTAT T CCT GGCTT - - ACT C- T GTTTTT TCTTCT GTTGTTAT T CCT GGCTT - - ACT C- TGTTTT T

A. eriantha

KP314044

WA002

A. cylindrica

KP314058

WA021

CCTC- CCCCCCTTGCT CCAATCC- - GCCT CTATCCGT CCTC- CCCCCCTTTTTCTAATCC- - GCCT CTATCT GT

TCTTCT GTTCT CATTCCTAGTTT - - ACT C- CGTTTTT TCTTCT GTTCTCAT T CCTAGTTT - - ACTC- CGTTTTT

TABLE 2 | The informative characters of nad2i3 and trnL-trnF sequences.

Absolute position mtDNA nad2i3 cpDNA trnL-trnF

1

$\begin{array}{lllllllllllllllllllllll}3 & 5 & 6 & 6 & 6 & 6 & 7 & 0 & 2 & 2 & 2 & 3 & 6 & 7 & 7 & 7 & 7 & 7 & 7 & 8 & 8\end{array}$

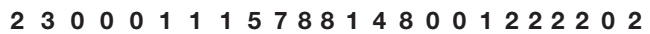

9778990007114520073890012386

A. fulvicoma

$\mathrm{NK} 01$

$\mathrm{NKO2}$

$\mathrm{NKO3}$

$C G A G A C A A C C \ldots G A C G C \ldots C G$

NKO4

NK05

$C G A G A C A A C C \ldots G A C G C \ldots G$

$C G A G A C A A C C \ldots G A C G C \ldots G G$

$C G A G A C A A C C$ - $A A C G C \ldots$

$C G A G A C A A C C \ldots G A C G C \ldots$

NK06

$\mathrm{NKO7}$

$C G A G A C A A C C$ - $A A C G C \ldots A G$

$C G A G A C A A C C$ - $A A C G C \ldots A G$

$\mathrm{NX07}$

NX08

$C G A G A C A A C C \ldots G A C G C \ldots G G$

$C G A G A C A A C C \ldots G A C G C \ldots C G$

NX09

NX10

NX10

$C G A G A C A A C C \ldots G A C G C \ldots C G$

$C G A G A C A A C C \ldots G A C G C \ldots C G$

$C G A G A C A A C C \ldots G A C G C \ldots C G$

A. cylindrica

OS17

WA021

A. eriantha

WA002

WA018

WA027

A T - - C C A C T TGACGC - . C C G

$A T \ldots$ - $A C T-T G A C G C \ldots G$

C G A G A A G T T T TAGAATGATTCA A

$C G A G A C A G T T T T A G A A T G A T T C A A$

$C G A G A C A G T T T T A G A A T G A T T C A A$

sequence differences between A. fulvicoma and A. eriantha that involved a 2-bp InDel and a 5-bp InDel at sites 284-285 and 719-723, respectively, and additional ten SNP sites. The nucleotide variation sites among Actinidia species in this study are shown in Table 2.

\section{The Data of SCNGs}

The four aligned SCNGs (1A, 2G, 2E, and 2C) in A. fulvicoma var. fulvicoma, A. fulvicoma var. lanata, A. cylindrica, and A. eriantha were 411 bp, 565 bp, 739 bp, and 532 bp, respectively. Among these SCNGs, there were 25, 16, and 10 informative characters in $1 \mathrm{~A}, 2 \mathrm{G}$, and $2 \mathrm{E}$, respectively, and 9 informative characters with a 3-bp InDel in 2C. In each SCNG, A. fulvicoma var. fulvicoma showed obvious informative character differences in various numbers of SNPs or InDel with either A. cylindrica or A. eriantha (Figure 3, Table S3). Similar to ITS, the four SCNGs suggested that neither $A$. cylindrica nor $A$. eriantha was the parent species of A. fulvicoma despite it being a hybrid species. Although most informative sites of these SNPs were synonymous mutations, the presence of species-specific non-synonymous amino acid sites in these functional genes supported that $A$. fulvicoma was an independent species different from $A$. cylindrica and $A$. eriantha. There was no sequence additivity from these two putative parental species found in A. fulvicoma. Similar nonsynonymous amino acid mutations could even distinguish the two varieties of $A$. fulvicoma, var. fulvicoma and var. lanata.

\section{Distribution of A. fulvicoma and Related Species}

A. fulvicoma is widely distributed in southern China (e.g. Guangdong, Guangxi, Jiangxi, and Hunan) and its type locality is Mount Luofu, Guangdong. A. cylindrica is endemic to north Guangxi, only existing in some counties, whereas A. eriantha is widely distributed in several provinces of China, such as Zhejiang, Fujian, Guangdong, Guangxi, and Jiangxi, according to previous studies (Cui, 1993; Qing and Liu, 2010). To investigate the distribution characteristics of A. fulvicoma and the two putative parental species, the distribution maps of three species are drawn in the main geographical overlapping areas of Guangdong and Guangxi (Figure 4). The distribution of $A$. fulvicoma, A. cylindrica, and A. eriantha is represented by yellow, pink, and green, respectively. The two putative parental species documented by Liu et al. (2017) are not sympatric in the type locality of A. fulvicoma, Mount Luofu, or even in the Guangdong province. Only a few overlapping areas of $A$. cylindrica and A. eriantha exist in the north of Guangxi.

\section{DISCUSSION}

\section{Multiple Lines of Evidence Reveal That A. fulvicoma Is Not a Hybrid Species}

Despite high similarity with one of putative parents identified by Liu et al. (2017), A. cylindrica or A. eriantha, in trnL-trnF and nad2-i3 sequences, the samples of A. fulvicoma possessed a unique haplotype that is distinct from those of the two putative parental species. Our results did not support the result of Liu et al. (2017), which suggested that A. cylindrica was the female parent of $A$. fulvicoma and A. eriantha the male parent. According to the inference of Liu et al. (2017), A. fulvicoma should have had A. 


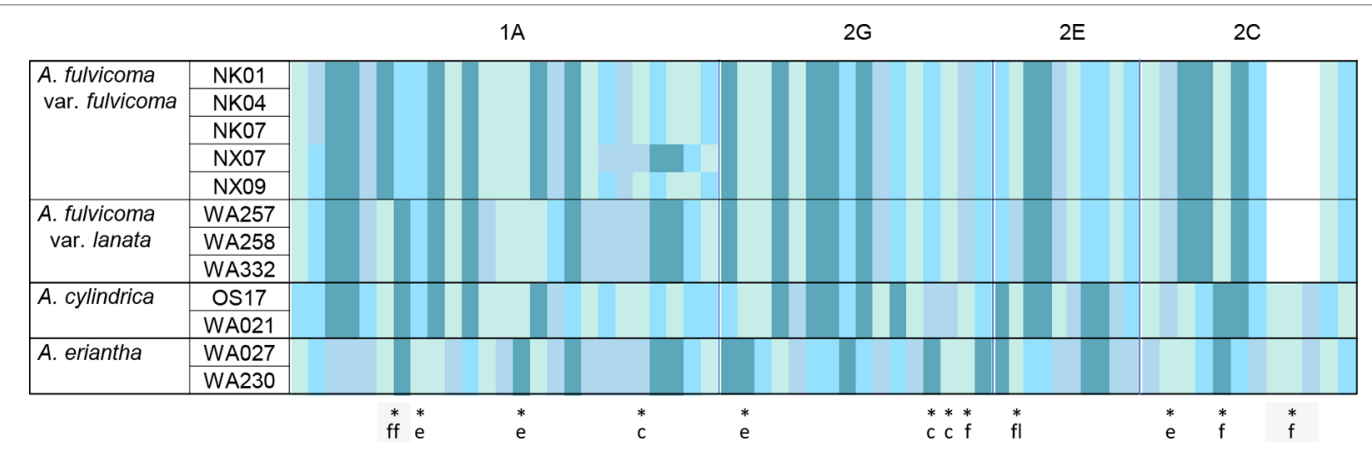

FIGURE 3 | The informative characters from sequences of four SCNGs (1A, 2G, 2E, and 2C) in A. fulvicoma, A. eriantha, and A. cylindrica. A. fulvicoma contained no genetic component from the two putative parents identified by Liu et al. (2017), $A$. eriantha, and $A$. cylindrica, suggesting that $A$. fulvicoma is an independent species, rather than a hybrid species. Gap is indicated in white, and four types of nucleotides are indicated in different colors. The corresponding species (or variety)specific amino acid sites are marked with an asterisk and the abbreviation of the taxon name (c, A. cylindrica; e, A. eriantha; f, A. fulvicoma; ff, A. fulvicoma var. fulvicoma, and fl, A. fulvicoma var. lanata).

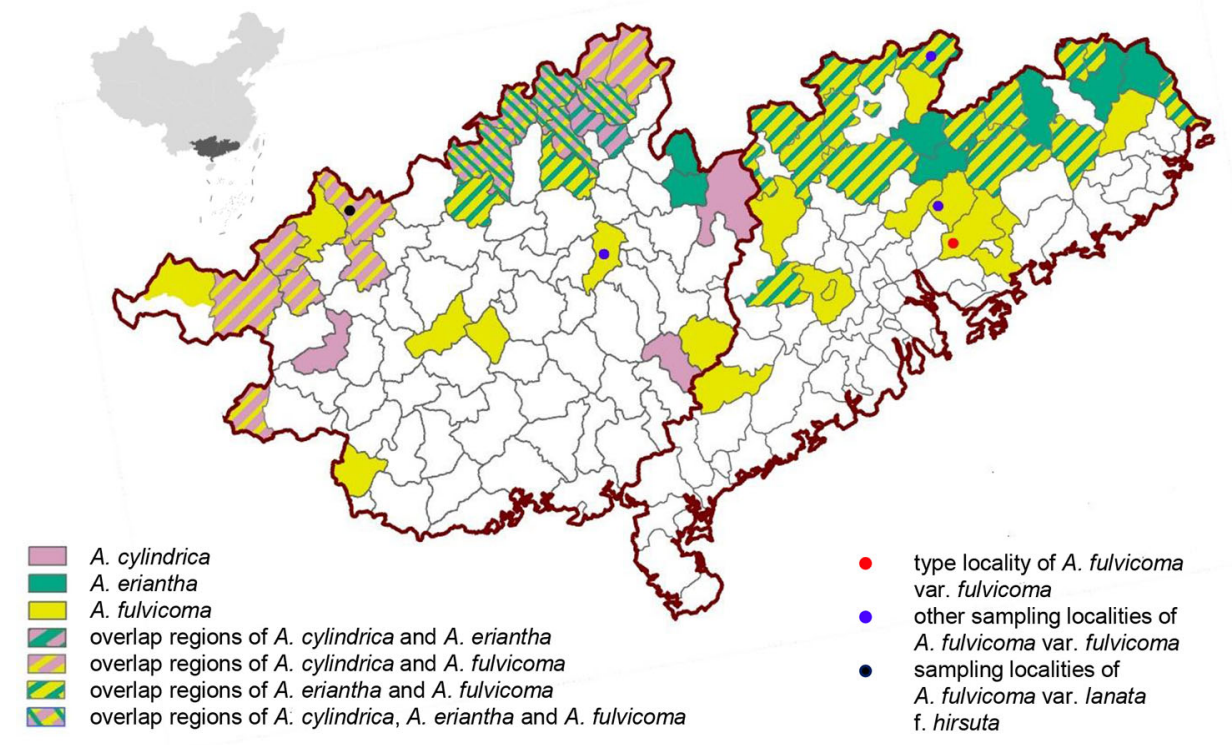

FIGURE 4 | The geographical distribution map of $A$. cylindrica, A. eriantha, and A. fulvicoma in Guangxi and Guangdong in China. The figure on the top left corner is a map of China showing the locations of Guangxi (left) and Guangdong (right) within China (black area). All counties with distribution records are shown in the map. The distribution areas of the three kiwifruit species are indicated by different colors (A. cylindrica, pink; A. eriantha, green; and A. fulvicoma, yellow), and their overlapping regions are shown by different types of stripes. The four sampling sites from different populations of $A$. fulvicoma are indicated by different colored dots.

cylindrica-type mtDNA nad2-i3 and A. eriantha-type cpDNA $\operatorname{trn} \mathrm{L}-\operatorname{trn} \mathrm{F}$, but this was not the case. A more critical piece of evidence from ITS phylogenetic analysis showed that no sequence of these two putative parental species and any other kiwifruit species existed in A. fulvicoma, let alone molecular additivities of informative characters of ITS sequences. A. fulvicoma occupied a monophyletic clade, which is far from either A. cylindrica or A. eriantha. Although ITS sequences in some flowering plant genera (e.g. Pyrus and Quercus) experience non-concerted evolution, the situation in A. fulvicoma is quite different. It has been proven that the high ITS polymorphism of Dayao Mountain population originated from intraspecific hybridization, being entirely unrelated to A. cylindrica and A. eriantha. All samples of $A$. fulvicoma from different populations form a monophyly. The informative characters from sequences of four SCNGs also showed that A. fulvicoma was an independent species that contains no genetic component from these two putative parental species. Thus, our data did not support that $A$. fulvicoma is a hybrid of A. cylindrica and A. eriantha.

Morphologically, the flower and fruit characteristics of Liu et al. (2017)'s sample were completely inconsistent with the original description of A. fulvicoma. Unlike their sample, all 
the samples that we collected from type locality and other places fell into the normal variation range of A. fulvicoma, and they did not present typical intermediate characteristics between $A$. eriantha and $A$. cylindrica. These morphological comparisons indicated that an incorrect sample led to error conclusion for the hybrid origin of A. fulvicoma in the study of Liu et al. (2017).

Furthermore, current distribution information also does not support the hybrid origin of A. fulvicoma. For the two putative parental species of Liu et al. (2017), A. cylindrica is endemic to Guangxi while A. eriantha is widely distributed in Fujian, Hunan, Guizhou, Guangdong, Guangxi, etc. However, type specimens of A. fulvicoma collected from Mount Luofu in Guangdong were outside of the natural distribution area of A. cylindrica, and, thus, they were impossible to hybridize with $A$. eriantha in the type locality. Although hybridization between the putative parental species might have occurred, as the result of sporadic contact long ago, for example, leaving no clear traces at present, ecological niche modeling showed that the decrease in the potential contact regions of two putative parental species since the Last Glacial Maximum (LGM) did not occur in Actinidia backbone taxa (Figure S20 in Liu et al., 2017). In general, Guangdong and Guangxi of South China are very suitable for the growth of different kiwifruit species. The extremely narrow distribution of $A$. cylindrica should not be the result of its area reduction or replacement of $A$. fulvicoma. Currently, there is no overlapping between $A$. cylindrica and A. eriantha in the entire Guangdong province, including the type locality of A. fulvicoma, neighboring regions, and most distribution areas in Guangxi. More importantly, none of our A. fulvicoma samples were of a hybrid origin between these two species.

Species misidentification and sampling error can lead to wrong conclusions of hybrid origins. In this study, multiple lines of evidence have confirmed that A. fulvicoma is an independent species, rather than a hybrid species. The sample of Liu et al. (2017) should be an interspecific hybrid between $A$. cylindrica and $A$. eriantha, probably collected from their overlapping regions in the north of Guangxi (Figure 3), but not real A. fulvicoma. Even if their sample of A. fulvicoma is a hybrid derived from $A$. cylindrica and $A$. eriantha, which is morphologically similar to A. fulvicoma, and is found in other localities, this cannot change the fact that A. fulvicoma from the type locality is not a hybrid. A. fulvicoma is still regarded as an independent species. However, if the samples from the type locality of the original variety of a species are hybrids, the nomenclature of other varieties of the species should be changed, according to the International Code of Botanical Nomenclature (ICBN). This will inevitably give rise to a series of taxonomic problems, but our evidence has shown that it is not the case.

\section{Niche Theory Involving Natural Hybridization and Species Distribution}

The colonization of ecological niches would be a dynamic process, with competition between parental lineages and hybrid populations, and hybridization could widen ecological adaptation (Choler et al., 2004). However, our conclusion that $A$. fulvicoma is a non-hybrid species has explicitly rejected the first prerequisite of the viewpoint of Liu et al. (2017) that the hybrid
A. fulvicoma should be a winner in the competition for ecological niches in comparison to one of its putative parents, A. cylindrica, with a very restricted distribution within its wide geographic range, further suggesting that hybridization can widen ecological adaptation (Choler et al., 2004). In the case of alpine Carex curvula, however, Choler et al. (2004) focused on two basically sympatric subspecies, subsp. rosae and subsp. curvula, and suggested that ecologically marginal populations of each subspecies are mainly composed of individuals with genotypes resulting from introgressive hybridization. The situation is obviously different from that of A. fulvicoma: it was identified by Liu et al. (2017) as a hybrid species of two distinct species (A. eriantha and A. cylindrica), and the distribution area of A. fulvicoma is much larger than that of A. cylindrica (Figure 4). New niche requirements and adaptations have also been found in the studies of invasive species. However, few cases involving species range expansion match the distribution comparison between A. fulvicoma and A. cylindrica. An exceptional case is Spartina anglica, which is originated from allopolyploidization (Baumel et al., 2002; Ainouche et al, 2004) rather than homoploid hybrid speciation, and its invasive success is largely depended on early artificial introduction. We expect to witness a case where the distribution of a species hybridized from two distinct homoploid species is much larger than that of one of its parent species, providing us a new understanding of the role of hybridization on species range expansion. It is not excluded that such examples can be found in other plants, however, whetherand to what extent-hybridization has an effect on the evolution of species ranges requires further study across more diverse taxa (Pfennig et al., 2016). Regardless of whether other hybrid species facilitate range expansion, at least in the case of A. fulvicoma, the fact that its geographical range is much larger than that of $A$. cylindrica is not caused by hybridization or introgression. Therefore, new evidence is needed to re-evaluate whether the hybrid species in kiwifruit occupy the large regions than their parent species because A. fulvicoma is identified by Liu et al. (2017) as the only interspecific hybrid species in Actinidia which distribution range is far larger than one of its parent species.

\section{The Implication for Future Practice on Genomic Studies of Hybrid Speciation}

Using conventional approaches, this study has provided unambiguous evidence that rejects the hybrid speciation hypothesis of a crucial kiwifruit species, A. fulvicoma, despite genomic evidence of frequent interspecific gene flow in kiwifruit. Considering that interspecific hybridization in flowering plants is relatively common (Rieseberg et al., 1995; Arnold, 1997) but homoploid hybrid speciation with strict standard is rare (Schumer et al., 2014), our case study of testing the hybrid origin hypothesis has highlighted that correct species identification is the premise and basis of hybrid speciation studies. In the taxonomic practice, similar incorrect species identification can be completely avoided but may still happen in the studies of other organisms. Observing hybrid ancestry in the genome of a few samples only provides direct evidence that they have an admixed genome (or even a genome of hybrid origin) but does not necessarily arrive at the optimistic conclusion 
of hybrid speciation (Schumer et al., 2018). Rigorous researchers tend to delimit the species boundaries using different approaches before identifying hybrid speciation events (e.g. Dejaco et al., 2016). In contrast, if an interspecific hybrid cannot be verified well, such results will mislead further studies of hybrid speciation. Therefore, our empirical approaches, presented here, have demonstrated the importance of the integration of morphological characters from type and non-type specimens, molecular data of multiple genes, and geographic information for correct species identification of putative interspecific hybrids. For the taxa involving frequent interspecific hybridization, we expect that extensive surveys of the putative hybrid and its possible parents, and multiple lines of evidence with strict criteria can be applicable to investigate a wider range of organisms, avoiding species misidentification and unreasonable judgment of hybrid speciation in further genomic analyses.

\section{DATA AVAILABILITY STATEMENT}

The datasets generated for this study can be found in GenBank, MK425065-MK425153; MK614167-MK614214.

\section{AUTHOR CONTRIBUTIONS}

JieY, HX, and YW collected plant materials. JieY performed the experiments, analyzed data, and wrote the first version of the

\section{REFERENCES}

Ainouche, M. L., Baumel, A., and Salmon, A. (2004). Spartina anglica C. E. Hubbard: a natural model system for analysing early evolutionary changes that affect allopolyploid genomes. Biol. J. Linn. Soc. 82, 475-484. doi: 10.1111/ j.1095-8312.2004.00334.x

Arnold, M. L. (1997). Natural Hybridization and Evolution (Oxford, UK: Oxford University Press).

Baumel, A., Ainouche, M., Kalender, R., and Schulman, A. H. (2002). Retrotransposons and genomic stability in populations of the young allopolyploid species Spartina anglica C. E. Hubbard (Poaceae). Mol. Biol. Evol. 19, 1218-1227. doi: 10.1093/oxfordjournals.molbev.a004182

Chat, J., Chalak, L., and Petit, R. J. (1999). Strict paternal inheritance of chloroplast DNA and maternal inheritance of mitochondrial DNA in intraspecific crosses of kiwifruit. Theor. Appl. Genet. 99, 314-322. doi: 10.1007/s001220051238

Chat, J., Jáuregui, B., Petit, R. J., and Nadot, S. (2004). Reticulate evolution in kiwifruit (Actinidia, Actinidiaceae) identified by comparing their maternal and paternal phylogenies. Amer. J. Bot. 91, 736-747. doi: 10.3732/ajb.91.5.736

Choler, P., Erschbamer, B., Tribsch, A., Gielly, L., and Taberlet, P. (2004). Genetic introgression as a potential to widen a species' niche: insights from alpine Carex curvula. Proc. Natl. Acad. Sci. U. S. A. 101, 171-176. doi: 10.1073/pnas.2237235100

Cipriani, G., Testolin, R., and Morgante, M. (1995). Paternal inheritance of plastids in interspecific hybrids of the genus Actinidia, revealed by PCRamplification of chloroplast DNA fragments. Mol. Gen. Genet. 247, 693-697. doi: 10.1007/bf00290400

Cipriani, G., Fiori, A., Moroldo, M., dePauli, P., Messina, R., and Testolin, R. (2003). Screening chloroplast, mitochondrial, and nuclear DNA sequences suitable for taxonomic studies in Actinidiaceae. Acta Hortic. 610, 337-342. doi: 10.17660/ActaHortic.2003.610.44

Coyne, J. A., and Orr, H. A. (2004). Speciation (Massachusetts, USA: Sinauer Associates). manuscript. WF, HX, ZS, WZ, and JiY analyzed the results and revised the manuscript. YW designed experiments, supervised the study, and co-wrote and revised the manuscript. All authors contributed to and approved the final manuscript.

\section{FUNDING}

This research was financially supported by the National Natural Sciences Foundation grants of China $(31870202,31370248)$ and the National Basic Research Program of China (973 programs, No. 2014CB954100).

\section{ACKNOWLEDGMENTS}

We thank the members of the Evolutionary Ecology Lab of Fudan University for useful discussions and critical comments on this manuscript.

\section{SUPPLEMENTARY MATERIAL}

The Supplementary Material for this article can be found online at: https://www.frontiersin.org/articles/10.3389/fgene.2019. 01250/full\#supplementary-material

Additional supporting information can be found online in the Supporting Information section at the end of the article.

Coyne, J. (1996). Speciation in action. Science 272, 700-701. doi: 10.1126/ science.272.5262.700

Cui, Z. X. (1993). Actinidia in China (Shangdong, China: Shandong Science and Technology Press). doi: 10.1093/sysbio/syw003

Dejaco, T., Gassner, M., Arthofer, W., Schlick-Steiner, B. C., and Steiner, F. M. (2016). Taxonomist's nightmare ... evolutionist's delight: An integrative approach resolves species limits in jumping bristletails despite widespread hybridization and parthenogenesis. Syst. Biol. 65, 947-974. doi: 10.1093/sysbio/syw003

Feliner, G. N., Alvarez, I., Fuertes-Aguilar, J., Heuertz, M., Marques, I., Moharrek, F., et al. (2017). Is homoploid hybrid speciation that rare? An empiricist's view. Heredity 118 (6), 513-516. doi: 10.1093/jhered/esi026

Gross, B. L., and Rieseberg, L. H. (2005). The ecological genetics of homoploid hybrid speciation. J. Hered. 96, 241-252. doi: 10.1093/jhered/esi026

Hovick, S. M., and Whitney, K. D. (2014). Hybridisation is associated with increased fecundity and size in invasive taxa: Meta-analytic support for the hybridisationinvasion hypothesis. Ecol. Lett. 17, 1464-1477. doi: 10.1111/ele.12355

Huang, H., and Liu, Y. (2014). Natural hybridization, introgression breeding, and cultivar improvement in the genus Actinidia. Tree Genet. Genomes 10, 11131122. doi: 10.1007/s11295-014-0771-8

Huang, S., Ding, J., Deng, D., Tang, W., Sun, H., Liu, D., et al. (2013). Draft genome of the kiwifruit Actinidia chinensis. Nat. Commun. 4, 2640. doi: 10.1038/ncomms3640

Kim, S. T., Sultan, S. E., and Donoghue, M. J. (2008). Allopolyploid speciation in Persicaria (Polygonaceae): Insights from a low-copy nuclear region. Proc. Natl. Acad. Sci. U.S.A. 105, 12370-12375. doi: 10.1073/pnas.0805141105

Larkin, M. A., Blackshields, G., Brown, N. P., Chenna, R., McGettigan, P. A., McWilliam, H., et al. (2007). Clustal W and Clustal X version 2.0. Bioinformatics 23, 2947-2948. doi: 10.1093/bioinformatics/btm404

Lee, J. Y., Mummenhoff, K., and Bowman, J. L. (2002). Allopolyploidization and evolution of species with reduced floral structures in Lepidium L. (Brassicaceae). Proc. Natl. Acad. Sci. U. S. A. 99, 16835-16840. doi: 10.1073/ pnas. 242415399 
Li, J., Huang, H., and Sang, T. (2002). Molecular phylogeny and infrageneric classification of Actinidia (Actinidiaceae). Syst. Bot. 27, 408-415. doi: 10.1043/ 0363-6445-27.2.408

Li, Z., Kang, M., Huang, H., Testolin, R., Jiang, Z., Li, J., et al. (2007). Phylogenetic relationships in Actinidia as revealed by nuclear DNA genetic makers and cytoplasmic DNA sequence analysis. Acta Hortic. 753, 45-58. doi: 10.17660/ ActaHortic.2007.753.3

Li, D., Qi, X., Li, X., Li, L., Zhong, C., and Huang, H. (2013). Maternal inheritance of mitochondrial genomes and complex inheritance of chloroplast genomes in Actinidia Lindl.: evidences from interspecific crosses. Mol. Genet.Genomics 288, 101-110. doi: 10.1007/s00438-012-0732-6

Liang, C., Chen, Y., and Wang, Y. (1984). “Actinidiaceae," in Flora Republicae Popularis Sinicae, vol. 195-301 . Ed. K. M. Fang (Beijing, China: Science Press), 309-334. doi: 10.1016/j.tree.2005.02.010

Liu, Y., Li, D., Zhang, Q., Song, C., Zhong, C., Zhang, X., et al. (2017). Rapid radiations of both kiwifruit hybrid lineages and their parents shed light on a two-layer mode of species diversification. New Phytol. 215, 877-890. doi: $10.1111 /$ nph.14607

Mallet, J. (2005). Hybridization as an invasion of the genome. Trends Ecol. Evol. 20, 229-237. doi: 10.1016/j.tree.2005.02.010

Mallet, J. (2007). Hybrid speciation. Nature 446, 279-283. doi: 10.1038/ nature 05706

Mayol, M., and Rosselló, J. A. (2001). Why nuclear ribosomal DNA spacers (ITS) tell different stories in Quercus. Mol. Phylogen. Evol. 29, 167-176. doi: 10.1006/ mpev.2001.0934

Mayr, E. (1963). Animal Species and Evolution (Massachusetts, USA: Harvard Univ. Press).

Pfennig, K. S., Kelly, A. L., and Pierce, A. A. (2016). Hybridization as a facilitator of species range expansion. Proc. R. Soc B.: Biol. Sci. 283, 20161329. doi: 10.1098/ rspb.2016.1329

Qing, H. N., and Liu, Y. (2010). A Checklist of Vascular Plants of Guangxi (Beijing, China: Science Press). doi: 10.1126/science.1086949

Rieseberg, L. H., and Wendel, J. F. (1993). "Introgression and its consequences in plants," in Hybrid Zones and the Evolutionary Process. Ed. R. G. Harrison (Oxford: Oxford University Press), 70-109. doi: 10.1038/s41437-017-0036-Z

Rieseberg, L. H., Vanfossen, C., and Desrochers, A. M. (1995). Hybrid speciation accompanied by genomic reorganization in wild sunflowers. Nature $375,313-$ 316. doi: $10.1038 / 375313 \mathrm{a} 0$

Rieseberg, L. H., Raymond, O., Rosenthal, D. M., Lai, Z., Livingstone, K., Nakazato, T., et al. (2003). Major ecological transitions in wild sunflowers facilitated by hybridization. Science 301, 1211-1216. doi: 10.1126/science.1086949

Rieseberg, L. H. (1997). Hybrid origins of plant species. Annu. Rev. Ecol. Syst. 28, 359-389. doi: 10.1146/annurev.ecolsys.28.1.359

Schumer, M., Rosenthal, G. G., and Andolfatto, P. (2014). How common is homoploid hybrid speciation? Evolution 68, 1553-1560. doi: 10.1111/evo.12399
Schumer, M., Rosenthal, G. G., and Andolfatto, P. (2018). What do we mean when we talk about hybrid speciation? Heredity 120, 379-382. doi: 10.1038/s41437017-0036-z

Stamatakis, A. (2006). RAxML-VI-HPC: maximum likelihood-based phylogenetic analyses with thousands of taxa and mixed models. Bioinformatics 22, 26882690. doi: 10.1093/bioinformatics/btl446

Taberlet, P., Gielly, L., Pautou, G., and Bouvet, J. (1991). Universal primers for amplification of three non-coding regions of chloroplast DNA. Plant Mol. Biol. 17, 1105-1109. doi: 10.1007/BF00037152

Tamura, K., Peterson, D., Peterson, N., Stecher, G., Nei, M., and Kumar, S. (2011). MEGA5: molecular evolutionary genetics analysis using maximum likelihood, evolutionary distance, and maximum parsimony method. Mol. Biol. Evol. 28, 2731-2739. doi: 10.1093/molbev/msr121

Testolin, R., and Cipriani, G. (1997). Paternal inheritance of chloroplast DNA and maternal inheritance of mitochondrial DNA in intraspecific crosses of kiwifruit. Theor. Appl. Genet. 94, 897-903. doi: 10.1007/s001220050493

White, T. J., Bruns, T., Lee, S., and Taylor, W. J. (1990). “Amplification and direct sequencing of fungal ribosomal RNA genes for phylogenetics," in PCR Protocols: A Guide to Methods and Applications. Eds. M. A. Innis, D. H. Gelfand, J. J. Sninsky and T. J. White (San Diego, USA: Academic Press), 315322. doi: 10.1002/j.1537-2197.1994.tb11475.x

Wolfe, A. D., and Elisens, W. J. (1993). Diploid hybrid speciation in Penstemon (Scrophulariaceae) revisited. Amer. J. Bot. 80, 1082-1094. doi: 10.1002/j.15372197.1993.tb15334.x

Wolfe, A. D., and Elisens, W. J. (1994). Nuclear ribosomal DNA restriction-site variation in Penstemon section Peltanthera (Scrophulariaceae): an evaluation of diploid hybrid speciation and evidence for introgression. Amer. J. Bot. 81, 1627-1635. doi: 10.1002/j.1537-2197.1994.tb11475.x

Wolfe, A. D., and Elisens, W. J. (1995). Evidence of chloroplast capture and pollenmediated gene flow in Penstemon section Peltanthera (Scrophulariaceae). Syst. Bot. 20, 395-412. doi: 10.2307/2419800

Zheng, X., Cai, D., Yao, L., and Teng, Y. (2008). Non-concerted ITS evolution, early origin and phylogenetic utility of ITS pseudogenes in Pyrus. Mol. Phylogen. Evol. 48, 89-903. doi: 10.1016/j.ympev.2008.05.039

Conflict of Interest: The authors declare that the research was conducted in the absence of any commercial or financial relationships that could be construed as a potential conflict of interest.

Copyright (c) 2020 Yang, Fu, Xu, Song, Zhang, Yang and Wang. This is an open-access article distributed under the terms of the Creative Commons Attribution License (CC $B Y$ ). The use, distribution or reproduction in other forums is permitted, provided the original author(s) and the copyright owner(s) are credited and that the original publication in this journal is cited, in accordance with accepted academic practice. No use, distribution or reproduction is permitted which does not comply with these terms. 